\title{
Frederick Banting et les possibilités de recherche pour les omnipraticiens
}

\author{
Iona Heath
}

$\mathbf{J}$ 'aurais bien aimé être là lorsque Banting s'est adressé à l'Association médicale canadienne en juin 1926. Je suppose qu'il y avait eu peu de femmes dans l'assistance et je me demande ce que l'auditoire avait pensé du jeune conférencier dont le curriculum vitae était déjà des plus extraordinaires et distingués. Le texte de sa conférence avait ensuite été publié dans le Journal de l'Association Médicale Canadienne en août de la même année.

Né en novembre 1891, Frederick Grant Banting avait alors à peine 34 ans. Trois ans auparavant, il était devenu le plus jeune lauréat du Prix Nobel de médecine pour sa participation à la découverte de l'insuline'. Immédiatement après avoir obtenu son diplôme de médecine en 1916, Banting s'était enrôlé dans le Corps de santé royal canadien. Il avait alors servi en France, avait été blessé lors de la bataille de Cambrai et, en 1919, avait reçu la Croix militaire pour héroïsme au combat. À son retour au Canada, il avait travaillé comme omnipraticien à London (Ont.) et avait étudié à la fois l'orthopédie et la pharmacologie avant de se lancer dans la recherche qui lui mériterait le Prix Nobel.

Lors de sa conférence, Banting a voulu « aborder le sujet de la recherche en médecine du point de vue de l'omnipraticien, espérant ainsi offrir des suggestions utiles à tous ceux qui sont ici présents aujourd'hui ». Ce qu'il a dit par la suite demeure en grande partie pertinent et utile presque 85 ans plus tard. En rendant hommage aux grands omnipraticiens-chercheurs de l'histoire, y compris Harvey, Sydenham, Addison et MacKenzie, Banting a signalé que « ces personnes étaient invariablement des travailleurs, des penseurs et des observateurs attentifs ».

Cette description ramène à l'essentiel un grand nombre des problèmes auxquels font face les omnipraticiens-chercheurs d'aujourd'hui. En raison de contraintes administratives ou des impératifs de la recherche moderne, il leur est de plus en plus difficile de combiner une carrière en recherche à la pratique de la médecine clinique, de sorte à appliquer la formule particulièrement productive de travail, de réflexion et d'observation mentionnée par Banting. En effet, ce dernier a affirmé que les grands omnipraticiens-chercheurs « observaient chaque signe et symptôme de la maladie et, en se fondant ensuite sur leur expé rience clinique, faisaient des déductions et élaboraient des théories ». Il se demandait par ailleurs si « aujourd'hui ... nous ne nous perdons pas parfois dans le labyrinthe des détails moins importants, en oubliant la question principale ». Si cela était déjà vrai en 1926, dans quelle mesure l'est-il encore davantage aujourd'hui, alors qu'il est à peu près impossible de résister à la tentation de trouver des associations à partir d'analyses de données effectuées par ordinateur, associations qui ne sont alors éclairées ni par l'expérience clinique ni par une théorie plausible de causalité ${ }^{2}$ ?

Banting a insisté sur l'importance d'une tenue méticuleuse des dossiers médicaux. « Lorsque l'on crée ces dossiers, ils peuvent paraître sans valeur, mais la mémoire est infidèle. Le dossier écrit est utile non seulement maintenant, mais pour toujours. » La difficulté à laquelle nous faisons face au début du XXI ${ }^{\mathrm{e}}$ siècle au Royaume-Uni, par exemple, est que nous avons maintenant des normes de qualité et des incitatifs financiers quant à la tenue de ces dossiers. Ces normes et incitatifs déterminent la façon dont ils sont tenus. Par ailleurs, les dossiers sont fondés sur des codes informatiques et sur notre compréhension actuelle des données probantes produites par la science biomédicale. Le problème est que de tels dossiers laissent peu de place à l'observation fortuite pouvant conduire à d'éventuelles connaissances futures. C'est pourquoi, en dépit des espoirs de Banting, il se peut que ces dossiers n'aient aucune pertinence pour l'avenir.
Intérêts concurrents :

Aucun déclaré

Cet article a été sollicité et n'a pas été soumis à

l'examen des pairs.

Correspondance :

Iona Heath,

iona.heath22@yahoo.co.uk

JAMC 2011. DOI:10.1503 /cmaj.110671 
Je me souviens de mon article favori sur le «patient mémorable », publié dans le British Medical Journal en $1997^{3}$. Il avait été soumis par un omnipraticien qui, alors qu'il était en train de résumer les dossiers de ses patients, avait trouvé le compte rendu d'une consultation remontant à plus de 10 ans. Le patient s'était présenté pour obtenir le renouvellement d'une ordonnance d'antibiotique contre sa rosacée et avait mentionné en passant que l'antibiotique en question l'aidait à digérer. Le médecin avait consigné une note : «Indigestion occasionnelle. Affirme que l'oxytet [oxytétracycline] la guérit ! » Dix ans plus tard, il écrivait : " Les propos d'un patient peuvent nous sembler bizarres, mais il se peut qu'il soit en train de nous révéler quelque chose de révolutionnaire. C'est à nos propres risques et périls que nous délaissons les éléments d'information ne correspondant pas à l'opinion-type. » Combien d'autres médecins ont entendu des patients leur transmettre des observations semblables au cours des années antérieures sans toutefois les consigner, laissant ainsi passer toute chance de découvrir Helicobacter pylori avant Barry J. Marshall et J. Robin Warren, qui, en 2005, ont obtenu le Prix Nobel de médecine pour leur découverte?

Déjà en 1926, Banting se plaignait du fait que les médecins s'en remettent de plus en plus aux tests cliniques au cours desquels, affirmait-il, ils " réfléchissent moins et font moins d'observations ». Le seul moyen d'étendre notre savoir est de douter de son étendue actuelle, de cultiver délibérément le doute - sentiment subjectif d'incertitude qui nous aide à circonscrire notre ignorance. Comme Banting le savait déjà grâce à son expérience d'omnipraticien, écouter nos patients peut nous aider à poursuivre nos réflexions, leurs anecdotes signalant la présence de lacunes et nous poussant à découvrir de nouveaux savoirs. En effet, non seulement ces anecdotes émanent-elles de l'écart entre un mot et son objet et explorent-elles l'écart entre ce qui est et ce qui aurait pu être et entre différentes vérités, mais elles constituent également un antidote perpétuel aux certitudes excessivement prévalentes de la médecine moderne.

En dépit de sa déclaration que « la recherche en médecine est possible même sans l'aide d'un laboratoire, car ses principes sont les mêmes à l'intérieur et à l'extérieur du laboratoire », Banting n'aurait pas pu découvrir l'insuline sans laboratoire. Depuis, l'écart entre la salle de consultation de l'omnipraticien et le laboratoire de physiologie ou de biochimie a continué à se creuser. Les omnipraticiens oeuvrant en milieu communautaire, et même la plupart des omnipraticiens en milieu universitaire, n'ont pas accès à de telles installations et n'ont aucun moyen de vérifier les théories qu'ils pourraient formuler à la suite de leurs observations et de leurs expériences.

Banting soutiendrait qu'il est essentiel d'essayer de refermer l'abîme entre la science médicale fondamentale et l'expérience clinique de tous les jours - et particulièrement la riche expérience longitudinale qui n'est trop souvent accessible qu'aux omnipraticiens. Au risque de m'exposer à un ridicule justifié, une de mes théories personnelles est que les symptômes de la ménopause produisent de façon périodique un épanchement bénéfique du système cardiovasculaire. Ce phénomène pourrait en partie expliquer le risque toujours plus faible de maladies cardiovasculaires chez les femmes en général ${ }^{4}$ et leur taux plus élevé chez celles qui suivent une hormonothérapie de remplacement ${ }^{5}$, cette dernière supprimant l'épanchement susmentionné. Je n'ai aucun moyen de tester cette théorie, mais quelqu'un pourrait peut-être le faire à ma place ou alors m'expliquer en quoi je me trompe.

Aujourd'hui, il est inconcevable, voire même ridicule, de penser qu'un jeune omnipraticien revenant d'une affectation en Afghanistan pourrait entreprendre des travaux de recherche aboutissant à un Prix Nobel. Pourquoi et comment pourrait-on rendre de nouveau accessibles les possibilités qui s'offraient à Banting ?

\section{Références}

1. Frederick G. Banting — biography. Stockholm (Suède) : la Fondation Nobel; 1923. Disponible à : http://nobelprize.org/nobel _prizes/medicine/laureates/1923/banting-bio.html (consulté le 11 mars 2011).

2. Davey Smith GD, Phillips AN. Confounding in epidemiological studies: why "independent" effects may not be all they seem. BMJ 1992;305:757-9.

3. Roscoe T. A memorable patient: early treatment of $\mathrm{H}$ pylori. BMJ 1997:315.

4. Ministère de la Santé. Health Survey for England 1998: cardiovascular disease. Londres (R.-U.) : The Stationary Office, 1999. www.archive.official-documents.co.uk/document/doh/survey98 /hse-02.htm\#2.3 (consulté le 13 mars 2011).

5. Humphries KH, Gill S. Risks and benefits of hormone replacement therapy: the evidence speaks. JAMC 2003; 168:1001-10.

Affiliation : Iona Heath est présidente du Royal College of General Practitioners du Royaume-Uni. 